\title{
Is there a relationship between the liver SUVmax values in FDG-PET/CT imaging and non-alcoholic fatty liver disease score?
}

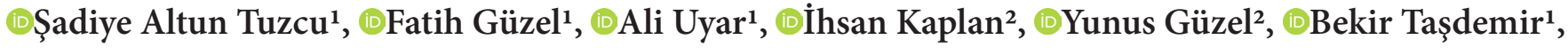 \\ Diondal Yalçın ${ }^{3}$ \\ ${ }^{1}$ Dicle University, Medical Faculty, Department of Nuclear Medicine, Diyarbakır, Turkey \\ ${ }^{2}$ Diyarbakır Gazi Yaşargil Research and Education Hospital, Department of Nuclear Medicine, Diyarbakır, Turkey \\ ${ }^{3}$ Dicle University, Medical Faculty, Department of Gastroenterology and Hepatology, Department of Internal Medicine, Diyarbakır, Turkey
}

Cite this article as: Altun Tuzcu Ş, Güzel F, Uyar A, et al. Is there a relationship between the liver SUVmax values in FDG-PET/CT imaging and non-alcoholic fatty liver disease score?. J Health Sci Med 2021; 4(6): 954-959.

\begin{abstract}
Aim: Non-alcoholic fatty liver disease is one of the most common causes of liver disease worldwide with an estimated prevalence of $20 \%-30 \%$ in adult population. Following the widespread utilization of PET in the evaluation of malignant diseases, F-18 FDG have also been reported to be used in non-malignant processes. The aim of this study is to elucidate whether the FDG SUVmax values determined by PET/CT in different adipose tissue samples and the liver change according to NAFLD score. During our desktop research we did not find any published article therefore, it is the first study in this field.

Materials and Method: A total of 230 patients who applied to Dicle University Faculty of Medicine, Department of Nuclear Medicine between March and April 2020 and who have been conducted FDG PET/CT for diagnosis, staging, restaging and evaluation of response to treatment were included in the study. Patients were divided into three groups according to their NAFLD score as patients with fibrosis score $<-1,455$ (the group in which severe fibrosis was excluded) as group-1, and those with NAFLD score between -1.455-0.676 (inter-mediate score) as group-2. and patients with a NAFLD score $>0.676$ (severe fibrosis group) group-3.

Results: Liver SUVmax levels were found to be significantly higher in group-3 than group-1. No significant difference was observed between group-2 and group-3. SUVmax levels measured from supracalvicular region, posterior scapular region and mesentery region were not different from each other in all three groups. Glucose-corrected liver SUVglu levels were found to be significantly lower in group-1 than group-3 ( $\mathrm{p}=0.001)$. In terms of liver SUVglu levels, group-1 and group-2 and group-2 and group-3 did not differ statistically from each other. Supracalvicular SUVglu, posterior scapular SUVglu and mesenteric SUVglu groups were not different from each other.

Conclusions: The most important result of this study could be elaborated with increased FDG uptake in NAFLD. Liver FDG uptake increases as the severity of NAFLD increases as demonstrated by the NAFLD score.
\end{abstract}

Keywords: SUVmax, FDG-PET/CT, nonalcoholic steatohepatitis score

\section{INTRODUCTION}

Non-alcoholic fatty liver disease (NAFLD) is one of the most common causes of liver disease worldwide. NAFLD is a broad terminology that includes simple fatty liver spectrum of chronic liver disease that progresses to non-alcoholic steatohepatitis (NASH), fibrosis, cirrhosis and end-stage liver (1-4). The excessive accumulation of triglycerides and cholesterol in the liver is classified as alcoholic fatty liver disease or non-alcohol-related fatty liver disease (NAFLD). NAFLD is the most common

chronic liver condition in the developed countries, with an estimated prevalence of $20 \%-30 \%$ in adult population $(5,6)$.

$\mathrm{PET} / \mathrm{CT}$ technology is a non-invasive imaging method commonly utilized as it provides important metabolic and functional data and high spatial resolution. Fluorine (F)-18, a radiopharmaceutical glucose analogue is a labeled Fluoro-2-deoxy-D-glucose (FDG) compound is mostly used in routine practice (7). The sensitivity and 
specificity of FDG PET/CT is quite high in metastatic liver lesions and other possible metastatic foci due to the whole body scanning advantage (8). Lymphocytes involved in infectious and inflammatory events, such as neutrophils and macrophages increased intracellular hexokinase and surface glucose transporter present a similar pattern to malignant cells and show affinity for F-18 FDG $(7,8)$.

Adenosine triphosphate located inside the inflammatory cells phosphorylates FDG and increases concentration cytoplasmic conversion of FDG to FDG 6-phosphate. However, as FDG-6-phosphate is not the convenient substrate for glucose-6-isomerase enzyme it cannot enter into metabolic reactions and accumulates inside the cells. The accumulation of FDG due to increased glucose transport activity during inflammatory processes on glucose metabolism and the amount of FDG accumulated derives information about the pathological areas showing impaired glucose metabolism in three dimensional display $(9,10)$.

SUV is a semiquantitative measurement which corresponds to measured activity normalized for body weight/surface area and injected dose $(11,12)$. The formula for calculation of SUV is region of interest (ROI) activity $(\mathrm{mCi} / \mathrm{mL}) \mathrm{x}$ body weight $(\mathrm{g}) /$ injected dose $(\mathrm{mCi})$. SUV is a proportional value without units, rather than being an absolute value in characterization of lesions, so for the quantification of tumour FDG uptake there was always a need for a site in the body which is presumed to have normal FDG uptake $(13,14)$. If the 18F-FDG uptake in the target lesion is greater than in the liver in terms of SUV, the hypermetabolic focus would be considered abnormal (15).

\section{Study Hypothesis}

The aim of this study is to elucidate whether the FDG SUVmax values determined by PET/CT in different adipose tissue samples of the liver and body change according to NAFLD score.

Fatty liver has been elaborated with different methods in previous studies. In this research we preferred to proceed by calculating the NAFLD score, considering it as a practical method. During our desktop research we did not find any published article therefore, it is the first study in this field.

\section{MATERIAL AND METHOD}

The study was carried out with the permission of Dicle University Medical Faculty Non-interventional Studies Ethics Committee (Date: 2021, Decision No: 358). All procedures were carried out in accordance with the ethical rules and the principles of the Declaration of Helsinki.
A total of 230 patients who applied to Dicle University Faculty of Medicine, Department of Nuclear Medicine between March and April 2020 and who have been conducted FDG PET/CT for diagnosis, staging, restaging and evaluation of response to treatment were included in the study. Because the study was designed retrospectively, no written Informed consent form was obtained.

NAFLD fibrosis score is a non-invasive, simple and easy-to-apply scoring system, which is obtained using 6 variable parameters (age, presence of impaired fasting glucose (IFG) or diabetes, body mass index (BMI), AST/ ALT ratio, platelet count, and albumin) and allows to accurately determine the presence, absence and severity of fibrosis(16). The files of the patients were examined (blood tests in the last month were examined), and the patients with ALT, AST, albumin and platelet levels in their files were included in the study. The height and weight of the patients were measured and their BMI (body mass index) was calculated. Patients were divided into three groups according to their NAFLD (Non-alcoholic fatty liver disease) score. According to the score defined by Angulo P et al. (16), patients with fibrosis score $<-1,455$ (the group in which severe fibrosis was excluded) as group-1, and those with NAFLD score between -1.455-0.676 (inter-mediate score) as group-2. and patients with a NAFLD score $>0.676$ (severe fibrosis group) were classified as group- 3 .

In group-1, a total of 28 of patients had diabetes or IGT and $66 \mathrm{did}$ not. In group-2, 37 had diabetes or IGT, while 50 did not. In group-3, 41 of the patients had diabetes or IGT, 8 did not.

SUVmax value was calculated by drawing $1 \mathrm{~cm}$ diameter ROI (region of interest) from the periphery of the right lobe of the liver. In addition, SUVmax values were measured from the fatty planes in the right supraclavicular region, from the subcutaneous tissue of the posterior part of the left scapula, and from the mesenteric area of the abdomen. All three groups were compared and was investigated whether there was a difference between the SUV values obtained from the supraclavicular region, especially the liver, and the mesentery of the infracalvicular region. In addition, patients' glucose-adjusted SUV values were SUVmax $\times$ blood glucose level/100 were calculated via SUVglu in order to compare 3 groups (17).

\section{Method and Device Information}

For the FDG PET/CT imaging, patients were required to fast for at least $6 \mathrm{~h}$ and have a blood glucose level of $140 \mathrm{mg} / \mathrm{dL}$. FDG at a dose of $0.1 \mathrm{mCI} / \mathrm{kg}$ was injected intravenously into the patients. After the injection, the patients were kept in a special lead-coated room for $1 \mathrm{~h}$ 
for the medication to spread through the whole body, and a CT scan of the whole body (from vertex to knees) was performed. Subsequently, whole-body emission scanning was performed with PET. A 2016 model Siemens Horizon brand PET/CT device with 3D-TOF was used for imaging. The slice thickness of the device was $3 \mathrm{~mm}$, and the images were created according to PET iterative and by the $\mathrm{CT}$ bp-LOR reconstruction processing method. The low-dose CT device used for anatomical detail and attenuation correction was adjusted to $80 \mathrm{~mA}$ and $120 \mathrm{kV}$ (Siemens Healthcare, GmbH Henkestrasse 127, 91052 Erlangen, Germany).

\section{Anthropometric Evaluations}

According to the height and body weight data obtained from the file records of the patients, the BMI of the patients was calculated with the formula weight(kg)/ height(m)2.

\section{Inclusion Criteria}

Patients who had blood tests in the last month and whose bio-distribution of F-18 FDG in the whole body was within normal limits.

\section{Exclusion Criteria}

Patients with liver cancer or liver metastasis were not included to the study as these cancer types may affect liver SUV. Patients with alcoholic stetohepatitis, viral hepatitis, pregnancy, autoimmune hepatitis, drug-induced toxic hepatitis, cirrhosis, insulin and pioglitazone using individuals have been excluded from the study. Imaging was performed in patients who received chemotherapy after waiting at least two weeks from the last chemotherapy

\section{Statistical Analysis}

IBM SPSS 21.0 for Windows statistical package program was used for the statistical evaluation of the data. Measurable variables were presented as mean \pm standard deviation, and categorical variables as numbers and percentages (\%). The chi-square $(\chi 2)$ test was used for comparing categorical variables. Oneway Anova and Post-Hoc Bonferroni were used for comparing measurable variables. Pearson correlation test was used to show correlation between NAFLD score and liverSUVmax, liverSUVglu age and glucose levels. A $\mathrm{P}$ value $\leq 0.05$ indicated a statistically significant difference.

\section{RESULTS}

The mean ages of all three groups were statistically different from each other. The mean age of group-1 was statistically significantly lower than group-2 and group-3 $(\mathrm{p}<0.001)$. The mean age was not different between group-2 and group-3. There was no difference between the groups in terms of gender. Group-1 mean body weight was significantly lower than group-2 $(p=0.03)$. There was no difference in body weight between group-2 and group- 3 .

There was no difference between the three groups in terms of height and BMI. Fasting blood glucose was found to be different from each other when all three groups were compared $(\mathrm{p}=0.001)$. Fasting blood glucose level was found to be significantly lower in group-1 than group-2 $(\mathrm{p}<0.0001)$.

The proportion of patients with impaired glucose tolerance and diabetes was found to be significantly higher in group-3 and group-2 than in group-1. Gender distribution, age, anthropometric parameters, glucose AST , ALT, albumin and platelet levels of the groups have been presented in Table 1.

\begin{tabular}{|c|c|c|c|c|}
\hline & $\begin{array}{c}\text { Group } 1 \\
(n=94)\end{array}$ & $\begin{array}{c}\text { Group } 2 \\
(n=87)\end{array}$ & $\begin{array}{c}\text { Group } 3 \\
(n=49)\end{array}$ & $\begin{array}{c}P \\
\text { value }\end{array}$ \\
\hline $\begin{array}{l}\text { Age } \\
\text { (years) } \pm S D\end{array}$ & $48.01 \pm 13.92$ & $59.5 \pm 13.3$ & $62.7 \pm 13.4$ & 0.0001 \\
\hline $\begin{array}{l}\text { Gender } \\
\text { (female/male) }\end{array}$ & $55 / 39$ & $40 / 47$ & $29 / 20$ & $>0.05$ \\
\hline $\begin{array}{l}\text { BMI } \\
(\mathrm{kg} / \mathrm{m} 2)\end{array}$ & $25.0 \pm 4.8$ & $26.7 \pm 4.7$ & $25.9 \pm 5.5$ & $>0.05$ \\
\hline $\begin{array}{l}\text { Glucose } \\
\text { (mg/dl) }\end{array}$ & $97.94 \pm 23.44$ & $106.6 \pm 21.16$ & $115.78 \pm 31.09$ & 0.001 \\
\hline $\begin{array}{l}\text { AST } \\
(\mathrm{IU} / \mathrm{L})\end{array}$ & $24.46 \pm 23,33$ & $27.5 \pm 18.5$ & $41.4 \pm 47.7$ & 0.004 \\
\hline $\begin{array}{l}\text { ALT } \\
(\mathrm{IU} / \mathrm{L})\end{array}$ & $23.11 \pm 22.87$ & $22.2 \pm 23.4$ & $21.3 \pm 23.9$ & $>0.05$ \\
\hline $\begin{array}{l}\text { Platelets } \\
\text { (cell/ml) }\end{array}$ & $331 \pm 109$ & $239 \pm 74$ & $170 \pm 95$ & 0.0001 \\
\hline $\begin{array}{l}\text { Albumin } \\
\text { (g/dl) }\end{array}$ & $3.89 \pm 0.56$ & $3.76 \pm 0.63$ & $2.99 \pm 0.75$ & 0.0001 \\
\hline
\end{tabular}

AST levels were significantly different from each other in the three groups $(\mathrm{p}=0.004)$. While the AST level of group-1 was found to be significantly lower than group-3 $(\mathrm{p}=0.003)$, the AST level of group- 2 was found to be significantly lower than group $-3(\mathrm{p}=0.02)$. ALT levels did not differ between groups.

All three groups were different from each other in terms of platelet levels. Group-1 had lower platelet levels than group-2, while group-2 had a significantly lower platelet level than group-3 $(\mathrm{p}=0.0001)$.

The albumin levels in the three groups were significantly different from each other $(\mathrm{p}=0.0001)$. While the albumin level of group-1 was found to be significantly lower than group -3 ( $p=0.0001)$, the AST level of group- 2 was found to be significantly lower than group-3 ( $\mathrm{p}=0.0001)$.

Liver SUVmax levels were found to be significantly higher in group-3 than group-1. No significant 
difference was observed between group-2 and group- 3 . SUVmax levels measured from supracalvicular region, posterior scapular region and mesentery region were not different from each other in all three groups. NAFLD score showed a positive correlation with the liver SUVmax $(\mathrm{r}=0.254, \mathrm{p}=0.02)$ liverSUVglu $(\mathrm{r}=0.284$, $\mathrm{p}=0.01)$, age $(\mathrm{r}=0.482, \mathrm{p}=0.001)$ and $(\mathrm{r}=0.225, \mathrm{p}=0.03)$ glucose levels. (Table 2, Figure 1).

Glucose-corrected liver SUVglu levels were found to be significantly lower in group-1 than group-3 $(\mathrm{p}=0.001)$. In terms of liver SUVglu levels, group-1 and group-2 and group-2 and group-3 did not differ statistically from each other. Supracalvicular SUVglu, posterior scapular SUVglu and mesenteric SUVglu groups were not different from each other (Table 2, Figure 2).

\begin{tabular}{|c|c|c|c|c|}
\hline & $\underset{(n=94)}{\text { Group } 1}$ & $\underset{(n=87)}{\operatorname{Group} 2}$ & $\begin{array}{c}\text { Group } 3 \\
(n=49)\end{array}$ & $\begin{array}{c}P \\
\text { value }\end{array}$ \\
\hline NAFLD score & $-2.80 \pm 1.18$ & $-0.447 \pm 0.643$ & $2.223 \pm 1.279$ & 0.0001 \\
\hline Liver SUVmax & $3.39 \pm 0.74$ & $3.66 \pm 0.73$ & $3.68 \pm 0.77$ & 0.02 \\
\hline $\begin{array}{l}\text { Supraclavicular } \\
\text { region SUVmax }\end{array}$ & $0.57 \pm 0.12$ & $0.53 \pm 0.12$ & $0.52 \pm 0.10$ & $>0.05$ \\
\hline $\begin{array}{l}\text { Posterior scapular } \\
\text { SUVmax }\end{array}$ & $0.55 \pm 0.12$ & $0.53 \pm 0.16$ & $0.55 \pm 0.15$ & $>0.05$ \\
\hline Mesenteric SUV & $0.65 \pm 0.36$ & $0.60 \pm 0.12$ & $0.61 \pm 0.15$ & $>0.05$ \\
\hline Liver SUVglu & $3.79 \pm 1.76$ & $4.50 \pm 1.85$ & $5.22 \pm 2.79$ & 0.001 \\
\hline $\begin{array}{l}\text { Supraclavicular } \\
\text { region SUVglu }\end{array}$ & $0.63 \pm 0.26$ & $0.65 \pm 0.29$ & $0.72 \pm 0.34$ & $>0.05$ \\
\hline $\begin{array}{l}\text { Supscapular } \\
\text { SUVglu }\end{array}$ & $0.61 \pm 0.30$ & $0.64 \pm 0.28$ & $0.75 \pm 0.34$ & $>0.05$ \\
\hline $\begin{array}{l}\text { Mesenteric } \\
\text { SUVglu }\end{array}$ & $0.25 \pm 0.18$ & $0.72 \pm 0.30$ & $0.86 \pm 0.45$ & $>0.05$ \\
\hline
\end{tabular}

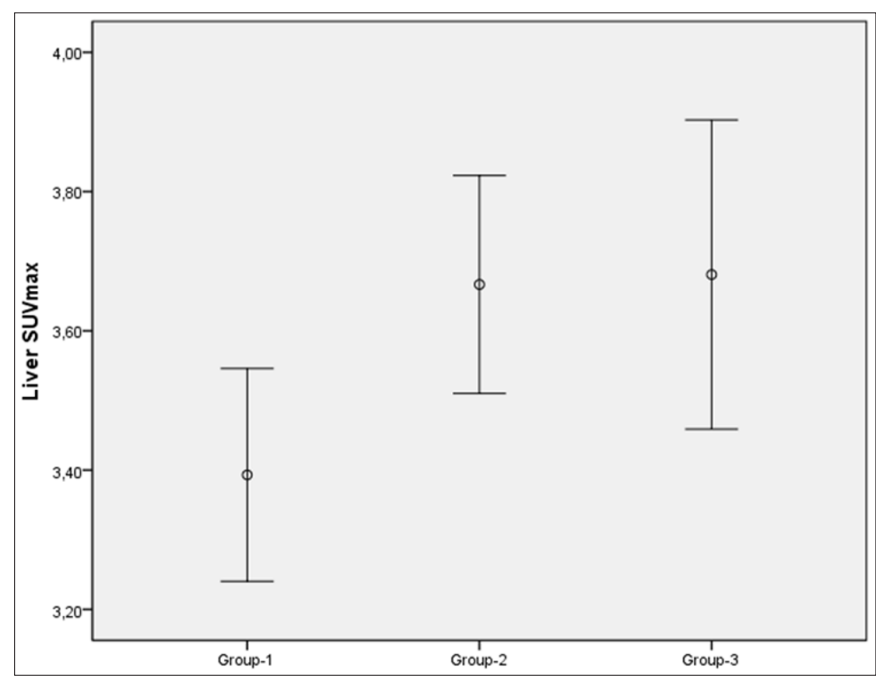

Figure 1. Liver SUVmax values of all three groups. The liver SUVmax value of group-1 was found to be significantly lower than group-3. Group-1 and group-2 Liver SUVmax values were not different. Liver SUVmax values of group-2 were not lower than group-3.

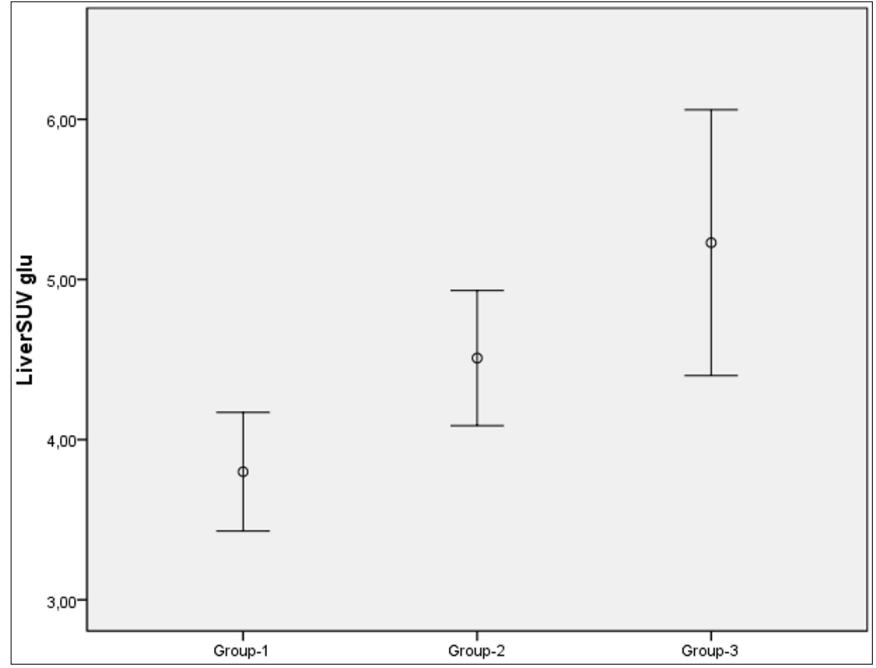

Figure 2. Liver SUVglu values of all three groups. The liver SUVglu value of group-1 was found to be significantly lower than group-3. Group-1 and group-2 Liver SUVglu values were not different. Liver SUVglu values of group-2 were not lower than group-3.

\section{DISCUSSION}

In our study, patients with a high NAFLD score have increased liver FDG Uptake. We did not find a similar study in our literature review. The articles in the literature on this subject are limited to FDG uptake measurements in patients with steatohepatitis, and fibrosis has been evaluated with either an invasive method such as liver biopsy or liver MRI, which is very expensive or invasive for the patient $(18,19)$. In our study, this easy and reproducible non-invasive model was used and its relationship with increased liver FDG uptake was emphasized.

The majority of patients with NAFLD will have fatty liver and liver inflammation resulting in altered hepatic FDG kinetics $(20,21)$. Fatty liver disease, which means accumulation of fat in the form of triglycerides and cholesterol in the liver cells, might induce some sort of inflammation and FDG uptake may be increased as a result of irreversible FDG accumulation in inflammatory cells, suggesting that FDG PET could be developed as a potential imaging approach to NASH (22). The reason for increased FDG uptake in fatty liver can be explained by increased Kupffer cell activity and FDG trapping (23).

In this study we found that liver SUV levels were found to be significantly higher in group-3 than group-1. No significant difference was observed between group-2 and group-3. SUV levels measured from supracalvicular region, posterior scapular region and mesentery region were not different from each other in all three groups. Glucose-corrected liver SUVglu levels were found to be significantly lower in group-1 than group-3. In terms of liver SUVglu levels, group-1 and group-2 and group-2 and group-3 did not differ statistically from each other. Supracalvicular SUVglu, posterior scapular SUVglu 
and mesenteric SUVglu groups were not different from each other. The most important phrase that one could derive from this data could be elaborated as, FDG uptake increased in NASH and FDG SUVmax has been detected statistically significantly higher in the group with higher NAFLD scores compared to the group with lower NAFLD scores. FDG uptake may be increased as a result of irreversible FDG accumulation in inflammatory cells, suggesting that FDG PET could be developed as a potential imaging approach to NASH $(24,25)$.

In this study fasting blood glucose was found to be different from each other when all three groups were compared. Fasting blood glucose level was found to be significantly lower in group-1 than group-2. Blood glucose levels can affect liver FDG SUVmax and therefore FDG SUVmax may have been different in the group with high NAFLD scores. FDG SUVglu levels, in which FDG uptake is calculated according to the patient's blood glucose level, can provide a correction opportunity according to glucose levels (26). In our study, FDG SUVglu levels were found to be significantly higher in the group with high NAFLD score. Based on these findings, the reason for the increase in FDG uptake seems to be due to a different cause than the patient's blood glucose levels.

The AST level of group-1 was found to be significantly lower than group-3, the AST level of group- 2 was found to be significantly lower than group-3. ALT levels did not differ between groups. All three groups were different from each other in terms of platelet levels. Group-1 had lower platelet levels than group-2, while group-2 had a significantly lower platelet level than group-3. The albumin levels of group-1 was found to be significantly lower than group-3. Ozulker et al. (27) found a statistically significant difference between the body weight, serum ALT levels, DM, and glucose levels of the patients with fatty liver and the control group. Patients with fatty liver disease have higher AST and ALT levels .

In some cases, NAFLD score may clarify the reason of increase in FDG uptake. Liver biopsy is performed to identify fibrosis and in addition to this the fibrosis score of patients can be predicted with lower FDG doses (28). In this case the calculation of the NAFLD score can reveal whether the FDG increase is due to the increase in the NAFLD score. The liver FDG SUV values are used as reference SUV values and if the NAFLD score is high in the patient, then the FDG SUVmax values will increase, thus causing false judgements because the reference SUV levels change. Currently liver biopsy is the gold standard in NASH to show inflammation, fibrosis and the severity of the disease. However, this procedure is invasive and has difficulties in routine implementation. In this study we have shown that a relationship can be established between FDG uptake and the severity of the disease (29).

\section{Limitations of the Study}

The main limitation of this study could be elaborated by its study population as all the enrolled individuals were cancer patients. One of the limitations of our study is that it had a retrospective nature and the mean age was different between the groups.

\section{CONCLUSION}

The most important result of this study could be elaborated with increased FDG uptake in NASH. Liver FDG uptake increases as the severity of NASH increases as demonstrated by the NAFLD score. An increase in NAFLD score causes the formation of the inflammatory pathway leading to fibrosis. Future prospective studies with larger number of patients where inflammatory markers are to be measured may enlighten this situation better.

\section{ETHICAL DECLARATIONS}

Ethics Committee Approval: The study was carried out with the permission of Dicle University Medical Faculty Non-interventional Studies Ethics Committee (Date: 2021, Decision No: 358).

Informed Consent: Because the study was designed retrospectively, no written informed consent form was obtained from patients.

Referee Evaluation Process: Externally peer-reviewed.

Conflict of Interest Statement: The authors have no conflicts of interest to declare.

Financial Disclosure: The authors declared that this study has received no financial support.

Author Contributions: All of the authors declare that they have all participated in the design, execution, and analysis of the paper, and that they have approved the final version.

\section{REFERENCES}

1. Schaffner F, Thaler H. Nonalcoholic fatty liver disease. Prog Liver Dis 1986: 8: 283-98.

2. Sheth SG, Gordon FD, Chopra S. Nonalcoholic steatohepatitis. Ann Intern Med 1997; 126: 137-45.

3. Matteoni CA, Younossi ZM, Gramlich T, et al. Nonalcoholic fatty liver disease: a spectrum of clinical and pathological severity. Gastroenterology 1999; 116: 1413-9.

4. Reid AE. Nonalcoholic steatohepatitis. Gastroenterology 2001; 121: 710-23.

5. Wah-Kheong C, Khean-Lee G. Epidemiology of a fast emerging disease in the Asia-Pacific region: non-alcoholic fatty liver disease. Hepatol Int 2013;7(1):65-71.

6. Papamiltiadous ES, Roberts SK, Nicoll AJ, et al. A randomised controlled trial of a Mediterranean Dietary Intervention for Adults with Non Alcoholic Fatty Liver Disease (MEDINA): study protocol. BMC Gastroenterol 2016; 16: 14. 
7. Parisi MT. Functional imaging of infection: conventional nuclear medicine agents and the expanding role of 18-F-FDG PET. Pediatr Radiol. 2011;41:803-10.

8. Israel $\mathrm{O}$, Keidar Z. PET/CT imaging in infectious conditions. Ann N Y Acad Sci 2011; 1228: 150-66.

9. European Association for the Study of the Liver (EASL); European Association for the Study of Diabetes (EASD); European Association for the Study of Obesity (EASO) EASLEASD-EASO Clinical Practice Guidelines for the management of non-alcoholic fatty liver disease. J Hepatol 2016; 64: 1388402 .

10. Lin CY, Ding HJ, Lin CC, et al. Impact of age on FDG uptake in the liver on PET scan Clin Imaging 2010; 34: 348-50.

11. Keramida G, Potts J, Bush J, Dizdarevic S, Peters AM. Hepatic steatosis is associated with increased hepatic FDG uptake.Eur J Radiol 2014; 83: 751-5.

12. Khandani AH, Wahl RL. Applications of PET in liver imaging .Radiol Clin North Am 2005; 43: 849-60.

13. Kumar R, Xiu Y, Yu JQ, et al. 18 F-FDG PET in evaluation of adrenal lesions inpatients with lung cancer. J Nucl Med 2004; 45: 2058-62.

14. van KouwenMC, Jansen JB, van Goor H, et al. FDG-PET is able to detect pancreatic carcinoma in chronic pancreatitis . Eur J Nucl Med Mol Imaging 2005; 32: 399-404.

15. Barrington NG, Mikhaeel L, Kostakoglu, et al. Role of imaging in thestaging and response assessment of lymphoma: consensus of theinternational conference on malignant lymphomas imaging working group. J Clin Oncol 2014; 32: 3048-58.

16. Angulo P, Hui JM, Marchesini G, et al. TheNAFLD fibrosis score: a noninvasive system that identifies liver fibrosis inpatients with NAFLD. Hepatology 2007; 45: 846-854.

17.Lee SM, Kim TS, Lee JW, Kim SK, Park SJ, Han SS. Improved prognostic value of standardized uptake value corrected for blood glucose level in pancreatic cancer using F-18 FDG PET. Clin Nucl Med 2011; 36: 331-6.

18. Wang G, Corwin M, Olson K, et al. Dynamic FDG-PET study of liver inflammation in non-alcoholic fatty liver disease. J Hepatol 2017; 66: 543-750.

19. Imajo K, Honda Y, Yoneda M, Saito S, Nakajima A. Magnetic resonance imaging for the assessment of pathological hepatic findings in nonalcoholic fatty liver disease. J Med Ultrason (2001). 2020; 47: 535-48

20. Hain SF, Curran KM, Beggs AD, et al. FDG-PET as a "metabolic biopsy" tool in thoracic lesions with indeterminate biopsy. Eur J Nucl Med 2001; 28: 1336-40

21. Beggs AD, Hain SF, Curran KM, O'Doherty MJ. FDG-PET as a "metabolic biopsy" tool in non-lung lesions with indeterminate biopsy. Eur J Nucl Med Mol Imaging 2002; 29: 542-6.

22. Keramida G, Potts J, Bush J, et al. Accumulation of (18)F-FDG in the liver in hepatic steatosis.AJR Am J Roentgenol. 2014; 203: 643-8. Erratum in: AJR Am J Roentgenol 2015; 204: 1137.

23. Kim YH, Kim JY, Jang SJ, et al. F-18 FDG uptake in focal fatty infiltration of liver mimicking hepatic malignancy on PET/CT images. Clin Nucl Med 2011; 36: 1146-8.

24. Le Y, Chen Y, Huang Z,Cai L, Zhang L.Intense FDG activity in focal hepatic steatosis.Clin Nucl Med 2014; 39: 669-72.

25. Han N, Feng H, Arnous MM, Bouhari A, Lan X.Multiple liver focal fat sparing lesions with unexpectedly increased (18)F-FDG uptake mimicking metastases examined by ultrasound (18) F-FDG PET/CT and MRI. Hell J Nucl Med 2016; 19: 173-5.

26. Qazi F, Oliver D, Nguyen N, Osman M. Fatty liver: impact on metabolic activity as detected with18F FDG-PET/CT. J Nucl Med 2008; 49: 263.

27. Ozulker T, Ozulker F, Assessment of the Effect of Fatty Infiltration on Hepatic FDG Uptake, Eur Arch Med Res 2019; 35: 27-32
28.Pak K, Kim SJ, Kim IJ, Kim K, Kim H, Kim SJ. Hepatic FDG Uptake is not Associated with Hepatic Steatosis but with Visceral Fat Volume in Cancer Screening. Nucl Med Mol Imaging 2012; 46: 176-81.

29. Bural GG, Torigian DA, Burke A, et al. Quantitative assessment of the hepatic metabolic volume product in patients with diffuse hepatic steatosis and normal controls through use of FDG-PET and MR imaging: a novel concept. Mol Imaging Biol 2010; 12: 233-9. 